\title{
Pathological Fracture Caused by Bone Metastasis of Rectal Cancer Misdiagnosed as a Common Fracture and Treated With Open Reduction and Internal Fixation: A Case Report
}

\section{Mingyang Yu ( $\nabla$ yumingyang1983@163.com )}

Affiliated Zhongshan Hospital of Dalian University

Yun Su

Affiliated Zhongshan Hospital of Dalian University

Shilong Zhao

Affiliated Zhongshan Hospital of Dalian University

Dongyi Li

Affiliated Zhongshan Hospital of Dalian University

Lin Guo

Affiliated Zhongshan Hospital of Dalian University

Fengde Tian

Affiliated Zhongshan Hospital of Dalian University

Ning An

Affiliated Zhongshan Hospital of Dalian University

Ruihu Hao

Affiliated Zhongshan Hospital of Dalian University

Changcheng Wang

Affiliated Zhongshan Hospital of Dalian University

Yao Zhang

Affiliated Zhongshan Hospital of Dalian University

\section{Case report}

Keywords: Rectal cancer, Bone metastases, Radiation therapy, Emission Computed Tomography, Open Reduction and Internal Fixation

Posted Date: November 23rd, 2020

DOl: https://doi.org/10.21203/rs.3.rs-111283/v1 
License: (c) (i) This work is licensed under a Creative Commons Attribution 4.0 International License. Read Full License 


\section{Abstract}

Background: Pathologic fractures following bone metastasis of rectal cancer are relatively uncommon.

Case presentation: A 59-year-old male rectal cancer patient in China with bone metastases in the right femur that caused a right femoral pathological fracture. According to emission computed tomography (ECT) and other examinations, and with the assistance of a multidisciplinary team, bone metastasis was diagnosed. Because the traumatic orthopedic surgeons did not notice his history of colorectal cancer preoperatively, there was no diagnosis of pathological fracture, and open reduction and internal fixation were performed. Additionally, there was no postoperative radiotherapy to the area or systemic treatment. Multiple re-examinations of the patient within 4 months after surgery revealed that the fracture did not heal. The patient was readmitted and treated 9 months after open reduction and internal fixation, and the tumor gradually spread in the proximal right femur.

Conclusions: The combination of a medical history, preoperative ECT examination, local DR examination, CT examination and MRI examination are necessary for a preoperative diagnosis. This case report mainly recorded the whole treatment and follow-up process.

\section{Background}

Metastatic carcinoma is the most common type of bone malignancy, and the skeleton is the third most common site for metastases, following the liver and lung ${ }^{[1,2]}$. The vast majority of bone metastases originate from cancers of the breast, prostate, and lung ${ }^{[3]}$. The incidence of bone metastases from colorectal cancer is not high ${ }^{[4]}$. There may be no specific symptoms in the early stage, and bone-related events will occur with the destruction of bone; the most serious result is pathological fracture ${ }^{[5]}$. Due to its relatively low incidence in patients with rectal cancer, bone metastasis is easily overlooked by orthopedic doctors. Here, we report a case of postoperative bone metastasis in a patient with rectal cancer and a pathological fracture in the proximal segment of the right femur. The patient underwent open reduction and internal fixation in accordance with conventional fracture treatment. No treatment for bone metastases of rectal cancer was performed, resulting in bone nonunion after 4 months and local tumor spread after 9 months. In this paper, we recorded the whole treatment process, provided the imaging data throughout the whole treatment process, and described the imaging changes after bone metastasis in detail.

\section{Ethics}

The experimental protocol was established according to the ethical guidelines of the Helsinki Declaration, and ethical approval for this study was provided by our university. The patient gave informed consent and consent for publication.

\section{Case Presentation}


A 59-year-old male patient was diagnosed with rectal cancer in January 2016 and underwent laparoscopic radical abdominoperineal resection of rectal cancer (Miles operation). The pathologic diagnosis after the operation was middle and low differentiated adenocarcinoma of the rectum; the whole layer of the tumor extended from the instrument to the serous membrane, there was no invasion of the nerves or vessels, no involvement of cancer in the distal and perioperative margins, sacral lymph nodes $(1 / 2)$ or inferior mesenteric lymph nodes $(1 / 2)$, and no lymph node metastatic carcinoma (1/2) in the intermediate group. Eleven cycles of chemotherapy with the FOLFOX6 regimen were started in February 2016. In June 2017, the SOX plan was changed to 8 cycles. Lung metastases were found in February 2018. Four cycles of chemotherapy with the FOLFIRI regimen were performed in February 2018. Liver metastases were found in October 2018. In July 2019, the right femur was fractured due to a fall, and the patient was admitted to the Department of Traumatology of our hospital (Fig. 1). After excluding contraindications, the orthopedic surgeon performed open reduction and internal fixation (Fig. 2). The wound healed, and the patient was discharged two weeks after the operation. Since bone metastasis of the tumor was not considered at that time, no radiotherapy or other antitumor treatments were performed in the postoperative area. The patient underwent digital radiography (DR) re-examinations every month after the operation, and four consecutive DR re-examinations were conducted (Fig. 3a-e). At the fourth postoperative review, the patient complained of obvious pain in the proximal right thigh. The patient was hospitalized again, and bone and soft tissue tumor surgeons in our hospital were consulted. Metastatic carcinoma in the right proximal femur was confirmed after reviewing the femur computed tomography (CT) and systemic bone images. Emission computed tomography (ECT) showed an enhanced radionuclide distribution in the right sacroiliac joint, right iliac bone and right femur (Fig. 4). Radiotherapy was then initiated for the right sacroiliac joint, the right iliac bone, and the right femur in combination with bevacizumab and bisphosphonates. Nine months after open reduction and internal fixation, the patient was unable to walk, and the pain in the proximal segment of the right femur was more pronounced. Upon re-examination, the tumor was found to be spreading in the proximal segment of the right femur and showed uncontrolled growth (Fig. 5). After communicating with the patient and his family, the patient's systemic condition was considered, so no operation was performed.

\section{Discussion And Conclusion}

The patient's comminuted fracture of the right proximal femur was seen by DR examination after his fall, but the DR examination did not provide effective pathological fracture information due to the overlap of the bone cortex at the broken end of the fracture. A retrospective analysis of the CT examination at that time revealed that the bone cortex of the proximal fracture of the right femur in the 2 o'clock to 8 o'clock directions presented osteolytic changes (Fig. 6). Unfortunately, this did not attract the attention of the doctor at that time, and the fracture was treated with open reduction and internal fixation (ORIF) according to the routine fracture care. Since pathologic fracture was not considered before the surgery, no soft tissue biopsy was performed during the surgery. Moreover, the intramedullary tumor tissue was not scraped when the fracture was fixed, and the surrounding soft tissue was not enlarged. We believe that this operation was successful simply from the perspective of achieving open reduction and internal 
fixation of the fracture. It can be seen that the position of the plate and screw internal fixation was good and the fracture reduction was satisfactory. The results of the subsequent four examinations showed that the fracture did not heal and the right femur had increased osteolytic destruction, and extensive abnormal osteogenesis in the proximal soft tissue of the right femur was also observed. Four months after the operation, ECT showed an abnormal concentration of radionuclides in the proximal segment of the right femur that was considered metastatic bone cancer. The patient did not undergo a histopathological examination of the proximal femur. ECT is highly sensitive but usually has a low specificity. The sensitivity of $99 \mathrm{mTc}$ scintigraphy has been reported to range from 62 to $89 \%$, with a falsepositive rate as high as $40 \%{ }^{6}$. However, combined with the patient's history, symptoms, CT examination, and joint consultation of oncologists, imaging physicians, orthopedic surgeons, and multidisciplinary physicians, the final diagnosis was bone metastasis. To be very clear, open reduction and internal fixation alone is not appropriate for the treatment of metastatic bone cancer ${ }^{7}$. Considering the patient's complex medical history and current health status, extensive tumor resection should be performed. The expanded resection should include the extent of bone resection, as the disease will invade the soft tissue around maximum resection margin, obtain a good surgical disease-free boundary, and include right femoral reconstruction with tumor prosthesis; additionally, postoperative radiotherapy should cover the entire area, scope and distal and proximal end of all tumor prostheses. Because pathologic fractures due to metastatic cancer were not considered at the time of surgery, the emergency department missed a very important magnetic resonance imaging (MRI) examination. Therefore, we have no way of knowing the extent of soft tissue invasion of the tumor at the time of the injury, and we have no way of determining the extent to which excision was possible to achieve a tumor-free surgical boundary. Subsequently, due to the lack of standard radiotherapy, we observed gradual local progression of the tumor during the following four months after surgery. However, radical right hip arthrotomy is not recommended because patients with liver, lung, and multiple lymph node metastases have lost the opportunity to undergo radical treatment of the tumor.

Bone metastases are an unfavorable consequence and portend poor survival, with a median survival duration of less than 6 months ${ }^{[4]}$. However, in all reports, the risk of bone metastasis from colorectal cancer is not high, so it is highly likely to be ignored by clinical orthopedic surgeons. In most patients, bone metastases will subsequently present bony destruction, the main mechanism of which is the release of a variety of cytokines by the tumor to increase the number of mononuclear cells followed by the secretion of RANKL to aggregate osteoclast mononuclear cells, thereby breaking the balance between local bone tissue osteogenesis/broken bone healing and leading to local osteolysis ${ }^{[6]}$. This bonedissolving effect not only provides more space and nutrients for the tumor but also causes hypercalcemia, bone pain and other symptoms. Since the stability of local bone is reduced, a low-energy injury may lead to pathological fracture of the bone with the lesion. Pathologic fractures occur in 10-30\% of all cancer patients, with proximal parts of the long bones being the most frequent fracture site, and femur fractures accounting for over half of all cases ${ }^{[7,8]}$ However, if a patient has both osteolytic and osteoblastic lesions, or if an individual metastasis has both osteolytic and osteoblastic components, then gastrointestinal cancers and squamous cancers maybe present ${ }^{[9]}$. 
Currently, it is believed that bone metastasis is not a direct cause of death in patients but rather leads to a series of complications caused by pathological fractures that seriously affects patient quality of life and significantly increases mortality ${ }^{[10]}$. A multidisciplinary approach should be decided by medical oncologists, radiotherapists, pain control teams, intervention radiologists, endocrinologists, orthopedic surgeons and psychologists. Finally, we recorded a pathological fracture caused by bone metastasis of rectal cancer based on the imaging findings. After conventional fracture treatment, a continuous process of bone nonunion and local tumor spread occurred. Due to the extreme rarity of the disease, it is the hope that this case will increase awareness of pathological fractures among traumatic orthopedic surgeons. The combination of a medical history, preoperative ECT examination, local DR examination, CT examination and MRI examination are necessary for a preoperative diagnosis.

\section{Abbreviations}

ECT:Emission Computed Tomography; DR:Digital Radiography; CT:Computed Tomography; MRI:Magnetic Resonance Imaging; ORIF:Open Reduction and Internal Fixation;RANKL:Receptor Activator of Nuclear Factor-k B Ligand.

\section{Declarations}

\section{Acknowledgments}

Not applicable.

\section{Authors' contributions}

MY and LG conceived the research idea and participated in its design; YS and NA contributed to the statistical analysis; $\mathrm{YZ}, \mathrm{RH}$ and FT participated in the data collection; SZ ,DL and CW contributed to the interpretation of the results and wrote the original draft. All authors have read and approved the final version of the manuscript, and agree with the order of presentation of the authors.

\section{Funding}

The Starting Fund for Doctor Talent Introduction of Affiliated Zhongshan Hospital of Dalian University (grant no. DLDXZSYY-BK201901).

\section{Availability of data and materials}

The datasets analysed in the current study are available from the corresponding author on reasonable request.

\section{Ethics approval and consent to participate}

Ethics approval was obtained from the Ethical Research Committee of Affiliated Zhongshan Hospital of Dalian University. All participants provided written informed consent before the commencement of the study. 


\section{Consent for publication}

Written informed consent was obtained from the patient's husband for publication of this case report and any accompanying images. A copy of the written consent is available for review by the Editor of this journal.

\section{Competing of interest}

The authors declare that they have no competing interests.

\section{Author details}

${ }^{1}$ Departments of Orthopaedics, Affiliated Zhongshan Hospital of Dalian University,City:Dalian, State:Liaoning, ZIP Code/Postal Code:116001, Country:China, Street Address:Jiefang Street, number 6.

${ }^{2}$ Department of Trauma Orthopaedic, Affiliated Zhongshan Hospital of Dalian University, City:Dalian, State:Liaoning,ZIP Code/Postal Code:116001, Country:China, Street Address:Jiefang Street, number 6. ${ }^{3}$ Department of Radiology, Affiliated Zhongshan Hospital of Dalian University, City:Dalian, State:Liaoning,ZIP Code/Postal Code:116001, Country:China, Street Address:Jiefang Street, number 6.

\section{References}

[1] Clines GA, Guise TA. Hypercalcaemia of malignancy and basic research on mechanisms responsible for osteolytic and osteoblastic metastasis to bone. Endocr Relat Cancer 2005;12: 549-83.

[2] Brook N, Brook E, Dharmarajan A, Dass CR, Chan A. Breast cancer bone metastases: pathogenesis and therapeutic targets. Int J Biochem Cell Biol 2018;96: 63-78.

[3] Coleman RE. Metastatic bone disease: clinical features, pathophysiology and treatment strategies. Cancer Treat Rev 2001;27: 165-76.

[4] Theriault RL, Theriault RL. Biology of bone metastases. Cancer Contro/ 2012;19: 92-101.

[5] Lunkiewicz M, Anderson S, Havakeshian S, Würzburg A. Practical input on bone tumor imaging: pathological fracture, risk features, and when to contact orthopaedics. Semin Musculoskelet Radiol 2019;23: 19-25.

[6] Macedo F, Ladeira K, Pinho F, Saraiva N, Bonito N, Pinto L, et al. Bone metastases: an overview. Oncol Rev 2017;11: 321.

[7] Chandrasekar CR, Grimer RJ, Carter SR, Tillman RM, Abudu A, Jeys LM, et al. Pathological fracture of the proximal femur in osteosarcoma: need for early radical surgery? ISRN Oncol 2012;2012: 512389.

[8] Selvaggi G, Scagliotti GV. Management of bone metastases in cancer: a review. Crit Rev Oncol Hemato/ 2005;56: 365-78. 
[9] Coleman RE, Seaman JJ. The role of zoledronic acid in cancer: clinical studies in the treatment and prevention of bone metastases. Semin Oncol 2001;28: 11-6.

[10] Alokaily F. Pathological fracture. Saudi Med J 2015 Jan;36(1):124-5.

\section{Figures}
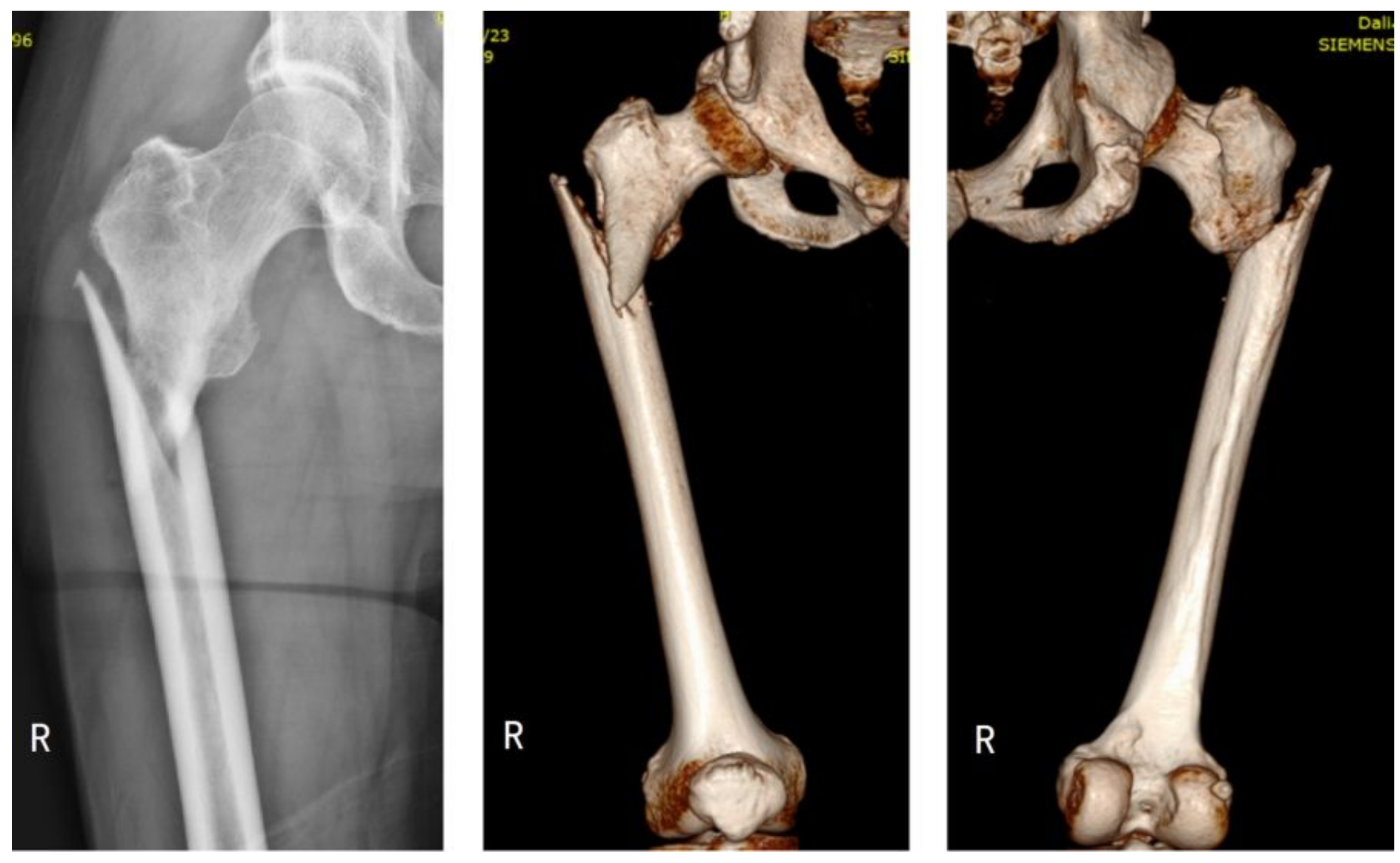

\section{Figure 1}

Since the greater trochanter and lesser trochanter of the patient's femur are intact, this fracture is not an intertrochanteric fracture but should be a proximal femoral shaft fracture. 

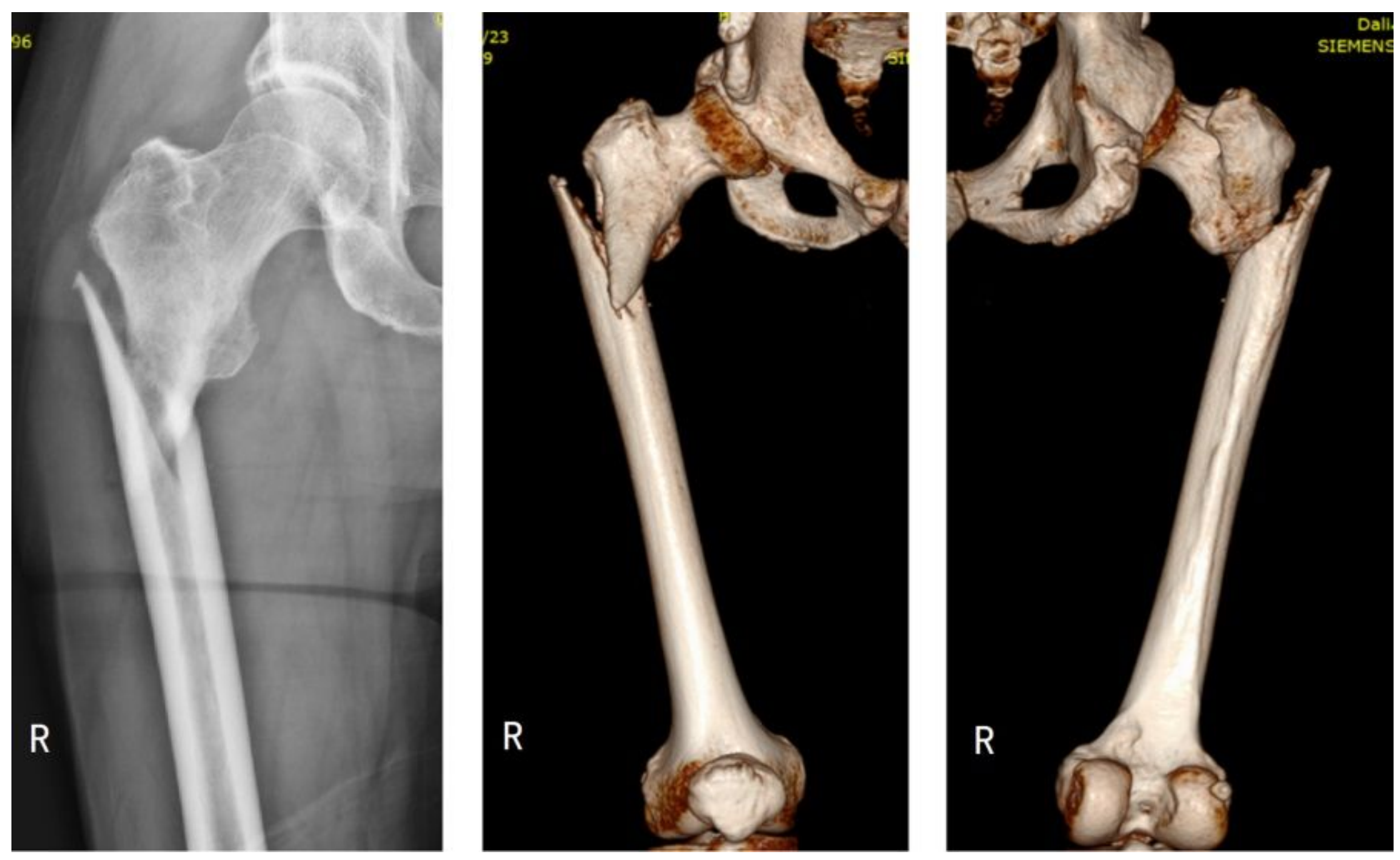

\section{Figure 1}

Since the greater trochanter and lesser trochanter of the patient's femur are intact, this fracture is not an intertrochanteric fracture but should be a proximal femoral shaft fracture. 

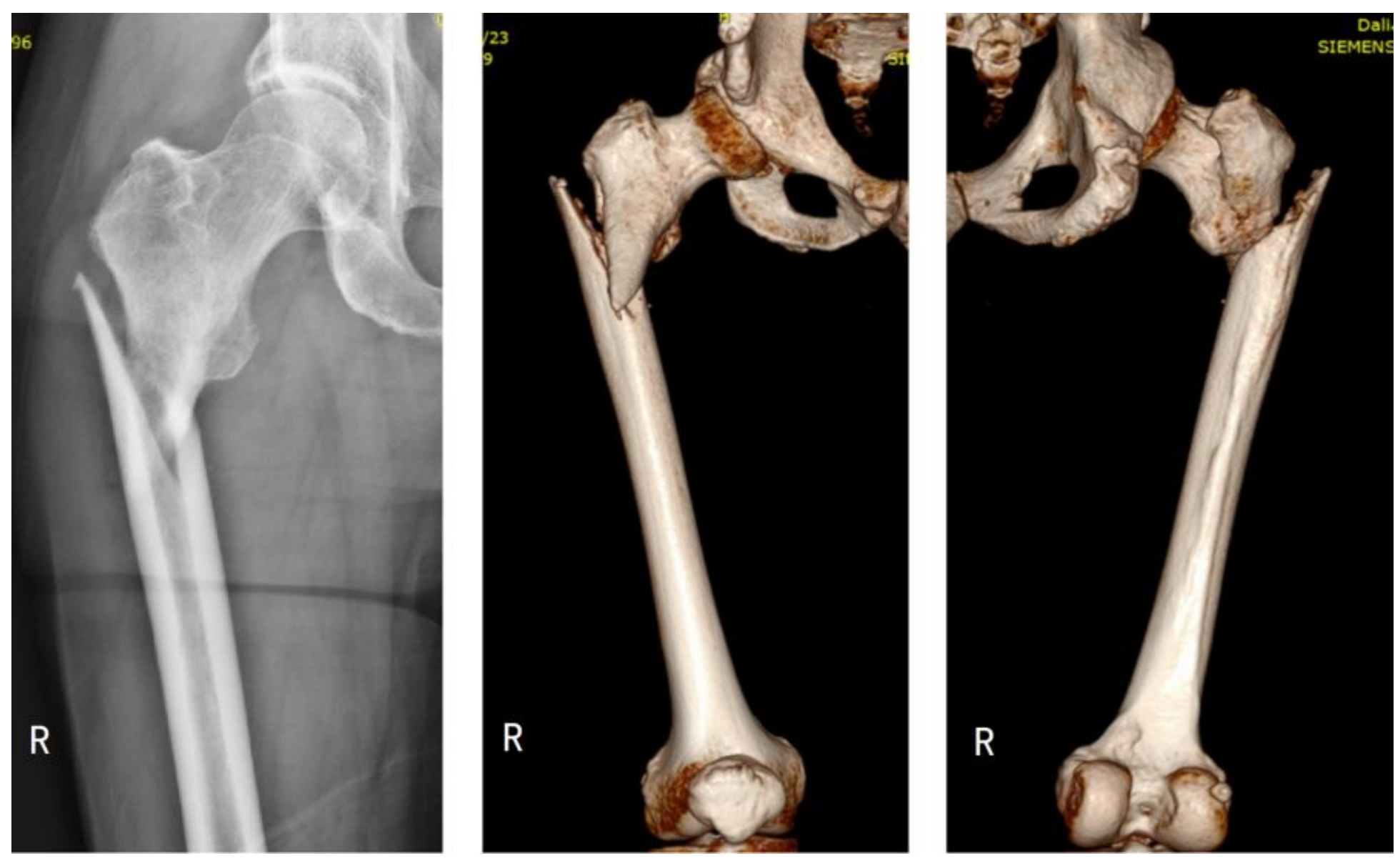

\section{Figure 1}

Since the greater trochanter and lesser trochanter of the patient's femur are intact, this fracture is not an intertrochanteric fracture but should be a proximal femoral shaft fracture. 

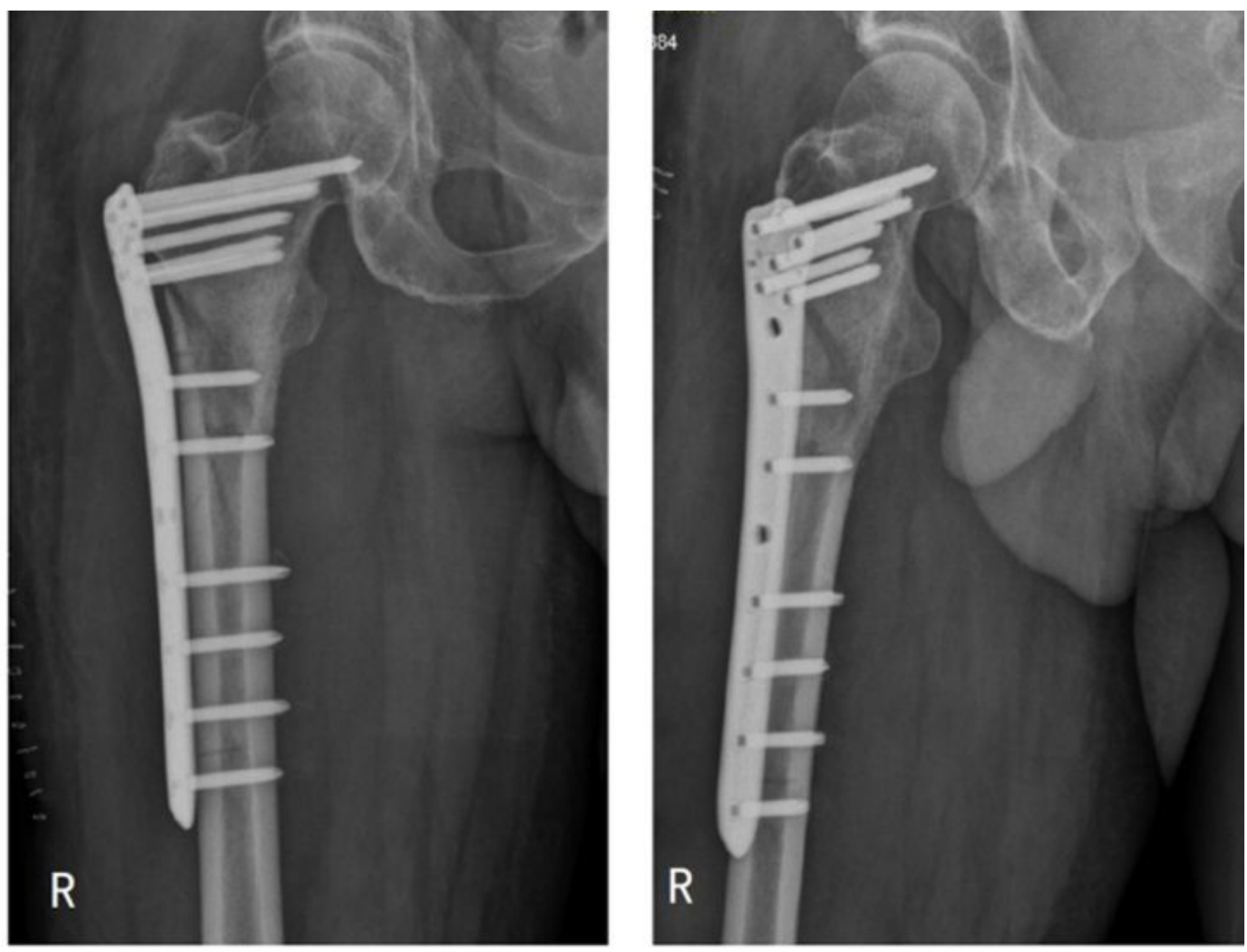

\section{Figure 2}

After open reduction and internal fixation, it can be seen that the fracture has achieved anatomic reduction, the continuity of the medial cortex of the femur shaft has been restored, the steel plate was placed in the right position, the number and layout of screws were reasonable, and the length was moderate. 

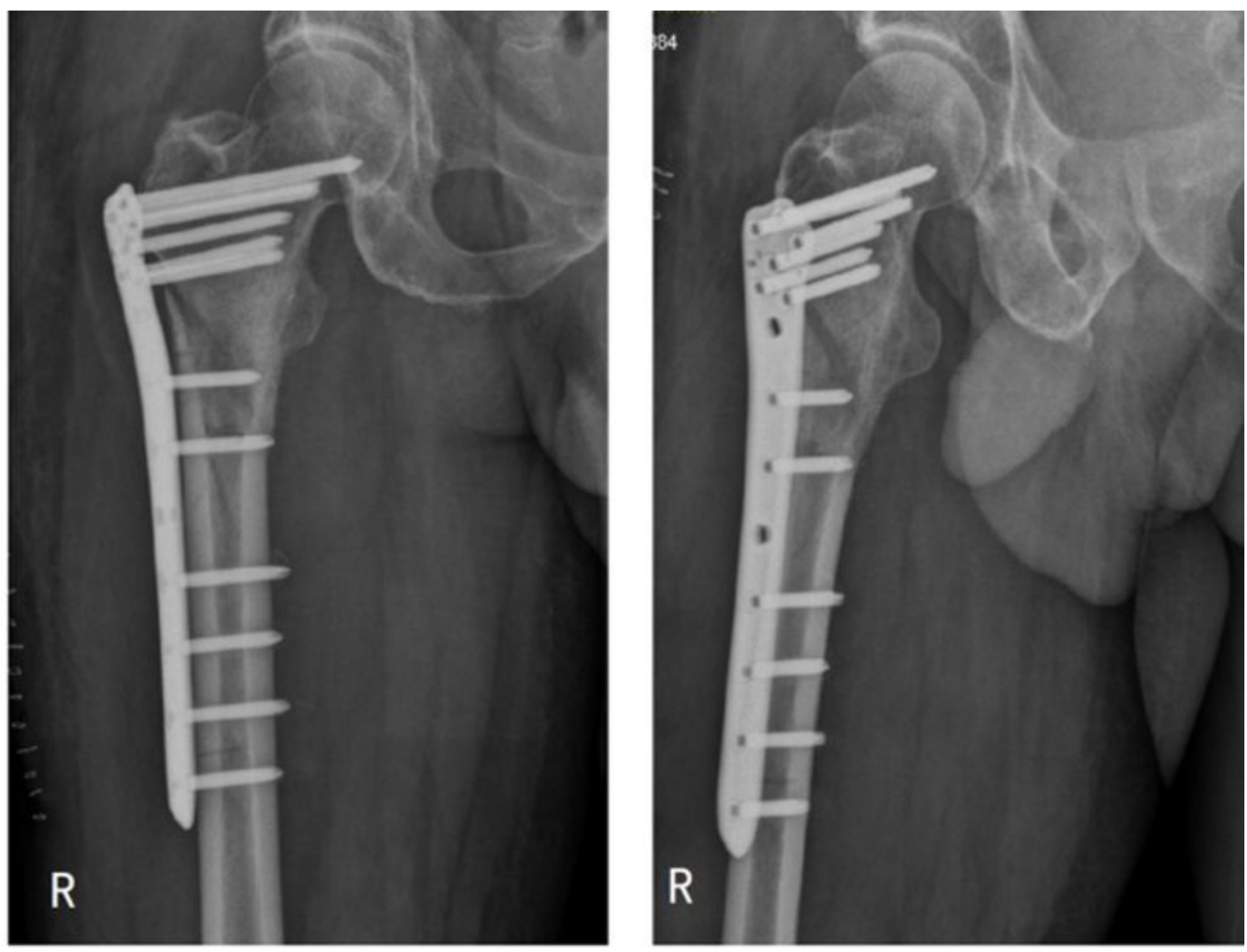

\section{Figure 2}

After open reduction and internal fixation, it can be seen that the fracture has achieved anatomic reduction, the continuity of the medial cortex of the femur shaft has been restored, the steel plate was placed in the right position, the number and layout of screws were reasonable, and the length was moderate. 

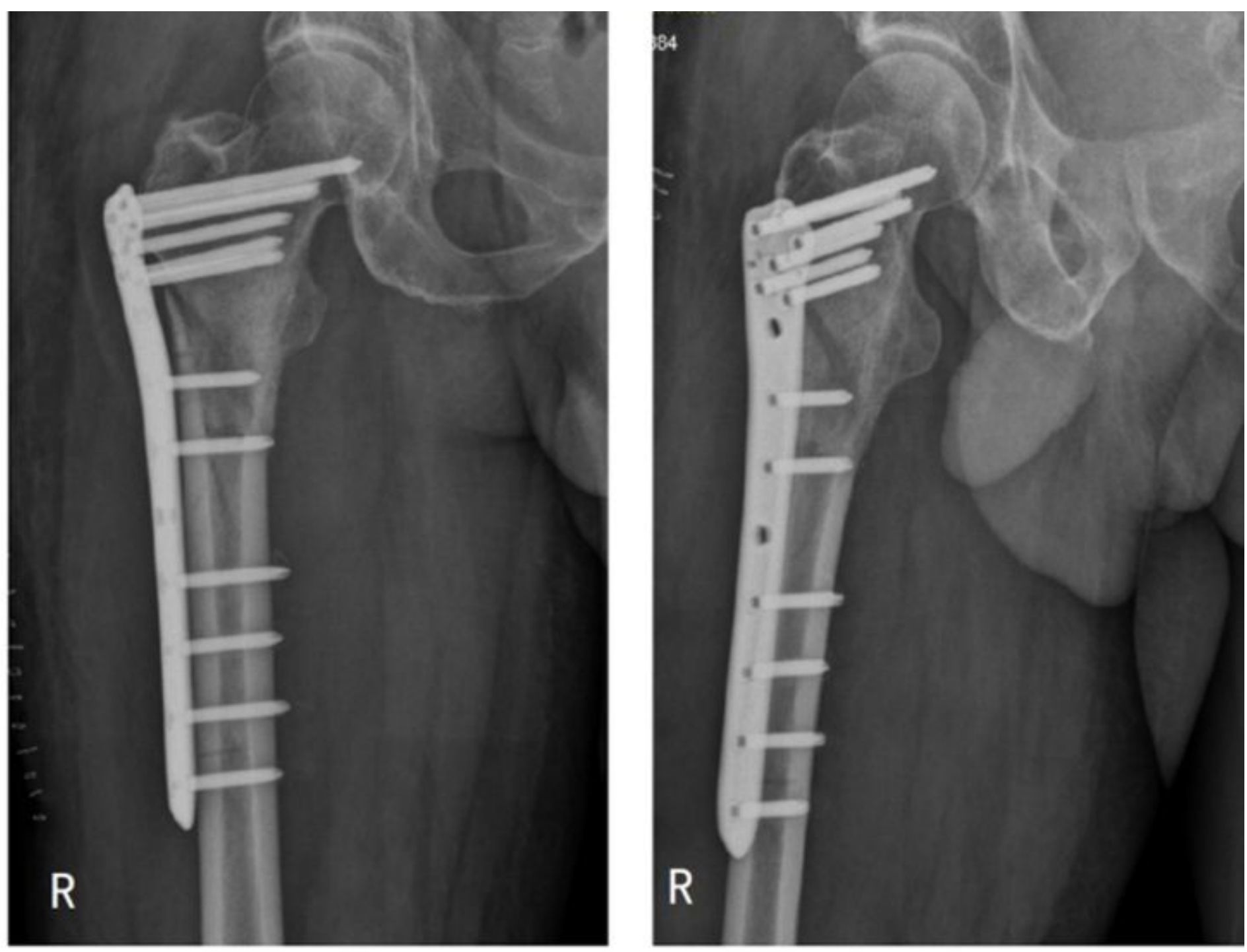

\section{Figure 2}

After open reduction and internal fixation, it can be seen that the fracture has achieved anatomic reduction, the continuity of the medial cortex of the femur shaft has been restored, the steel plate was placed in the right position, the number and layout of screws were reasonable, and the length was moderate. 

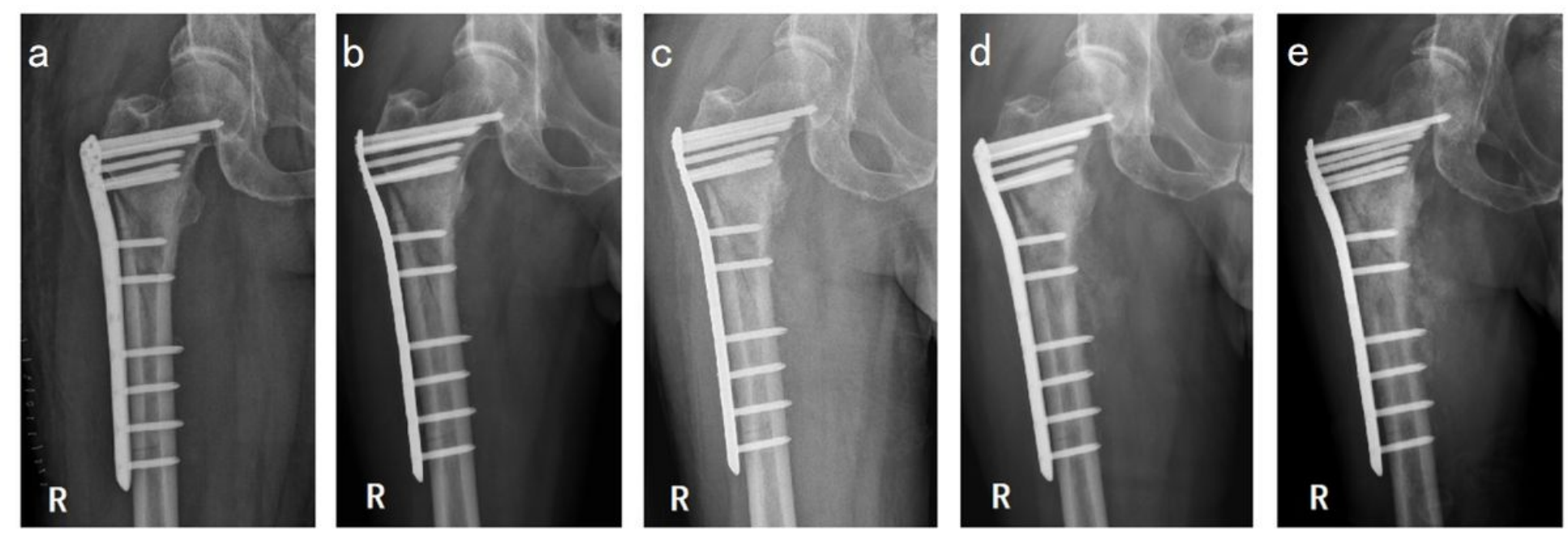

\section{Figure 3}

The patient was followed up from the day after surgery to the 4th month after surgery. The fracture site achieved anatomic reduction after surgery, the steel plate was in the right position, and the screw layout and lengths were reasonable (Fig. 3a) No callus formation was found in the first month (Fig. 3b), and from the second month, the medial femur shaft began to show osteolytic changes, and the lesser trochanter showed osteolytic changes (Fig. 3c). By the third month, this change was clearly evident (Fig. $3 \mathrm{~d}$ ); at the beginning of the fourth month, there was significant ectopic osteogenesis around the fracture line (Fig. 3e).
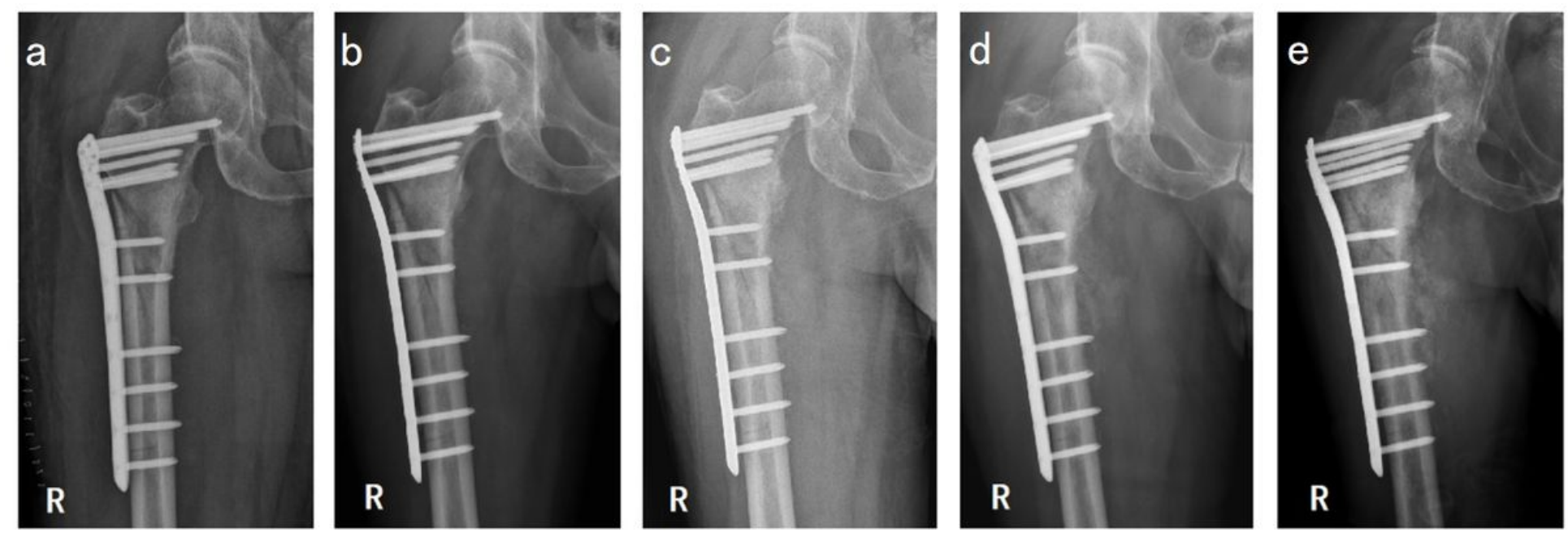

\section{Figure 3}

The patient was followed up from the day after surgery to the 4th month after surgery. The fracture site achieved anatomic reduction after surgery, the steel plate was in the right position, and the screw layout and lengths were reasonable (Fig. 3a) No callus formation was found in the first month (Fig. 3b), and from the second month, the medial femur shaft began to show osteolytic changes, and the lesser trochanter showed osteolytic changes (Fig. 3c). By the third month, this change was clearly evident (Fig. 
$3 d$ ); at the beginning of the fourth month, there was significant ectopic osteogenesis around the fracture line (Fig. 3e).
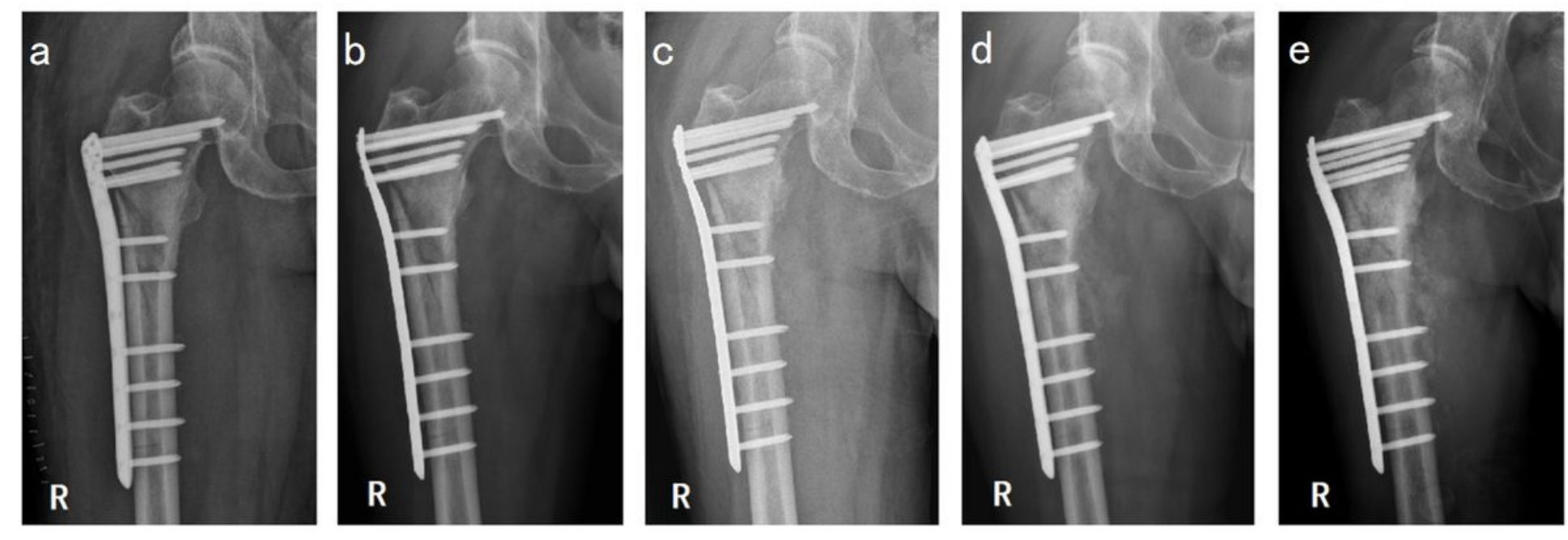

\section{Figure 3}

The patient was followed up from the day after surgery to the 4th month after surgery. The fracture site achieved anatomic reduction after surgery, the steel plate was in the right position, and the screw layout and lengths were reasonable (Fig. 3a) No callus formation was found in the first month (Fig. 3b), and from the second month, the medial femur shaft began to show osteolytic changes, and the lesser trochanter showed osteolytic changes (Fig. 3c). By the third month, this change was clearly evident (Fig. $3 d$ ); at the beginning of the fourth month, there was significant ectopic osteogenesis around the fracture line (Fig. 3e). 


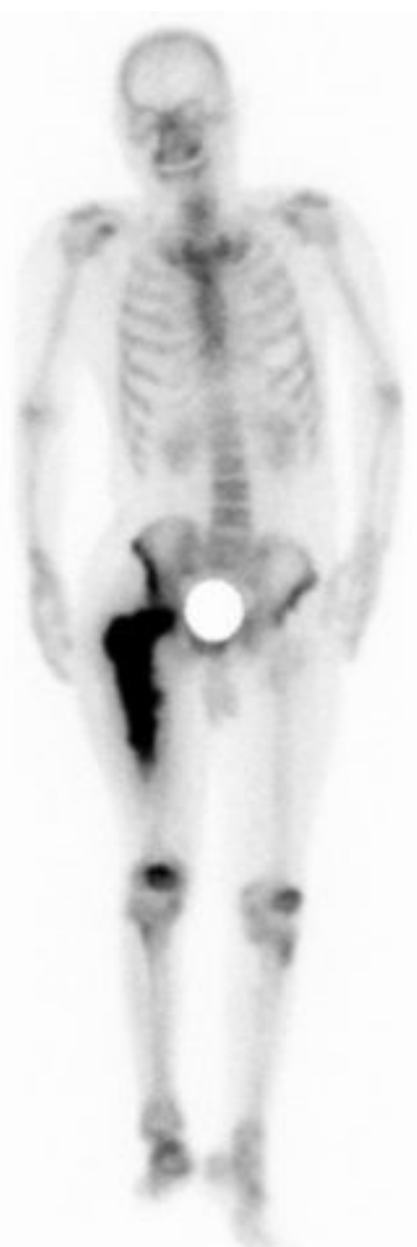

Anterior

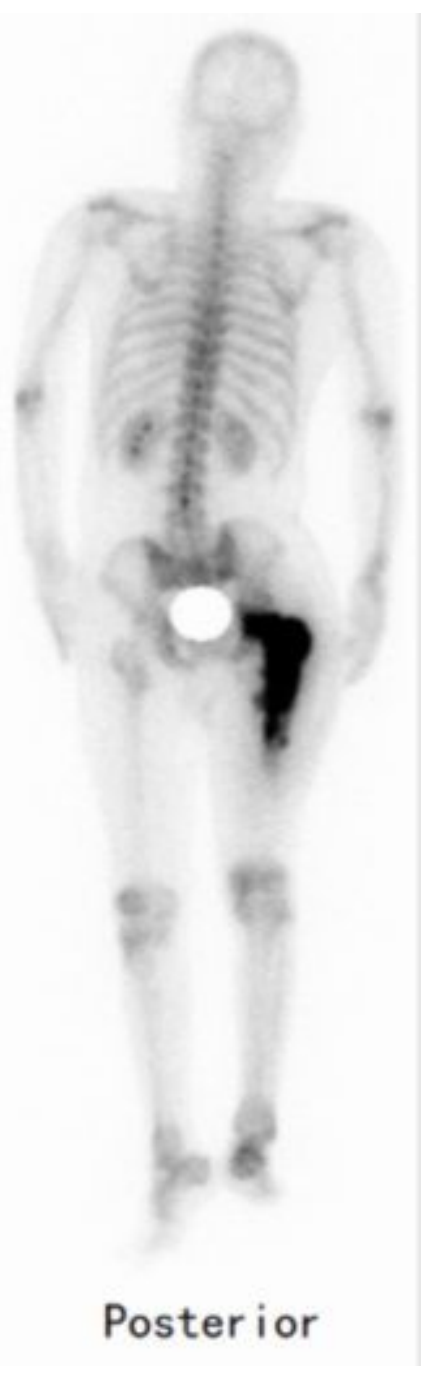

Figure 4

Systemic bone imaging was performed 4 months after surgery. The results showed an abnormal concentration of radionuclides in the proximal region of the right femur, which was considered tumor bone metastasis. 


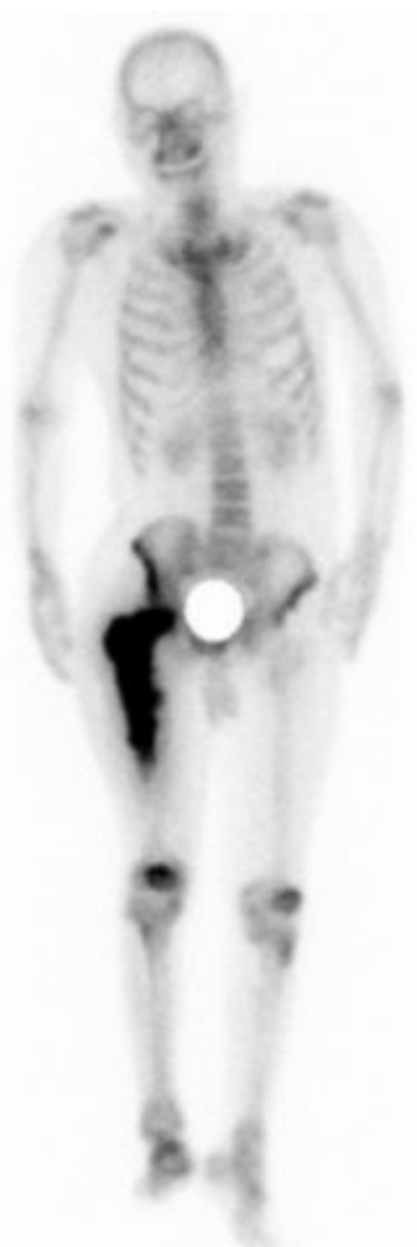

Anterior

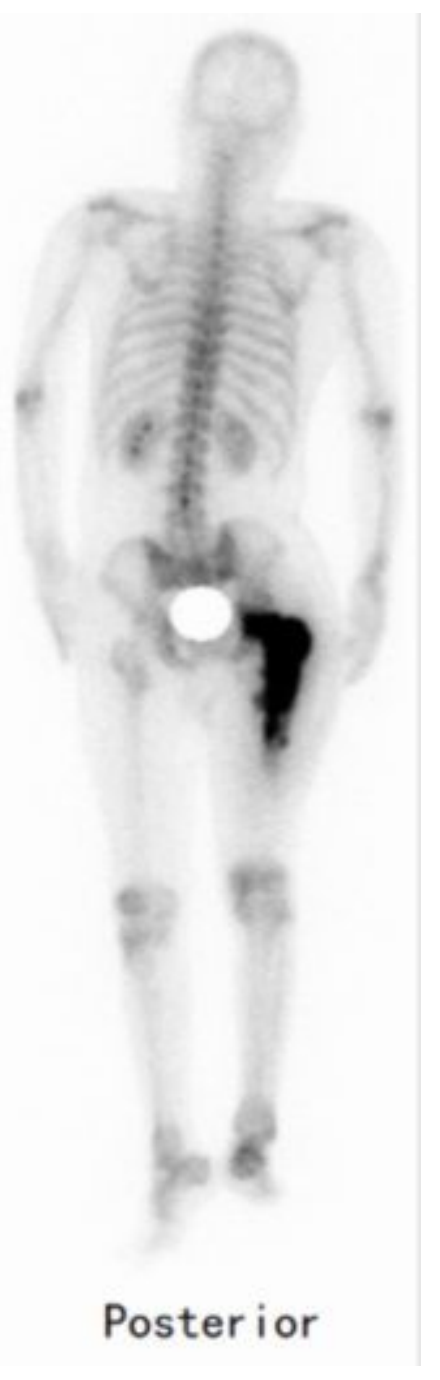

Figure 4

Systemic bone imaging was performed 4 months after surgery. The results showed an abnormal concentration of radionuclides in the proximal region of the right femur, which was considered tumor bone metastasis. 


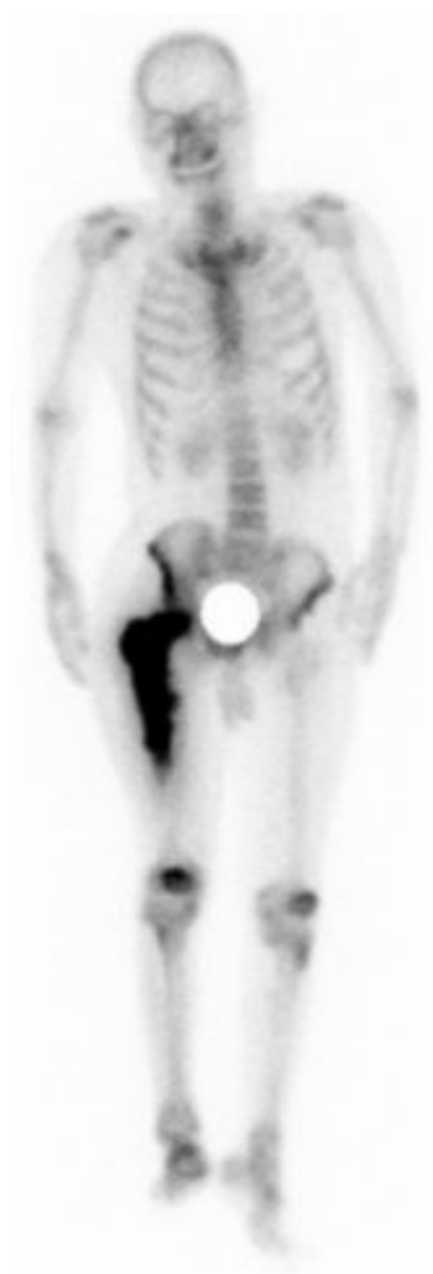

Anterior

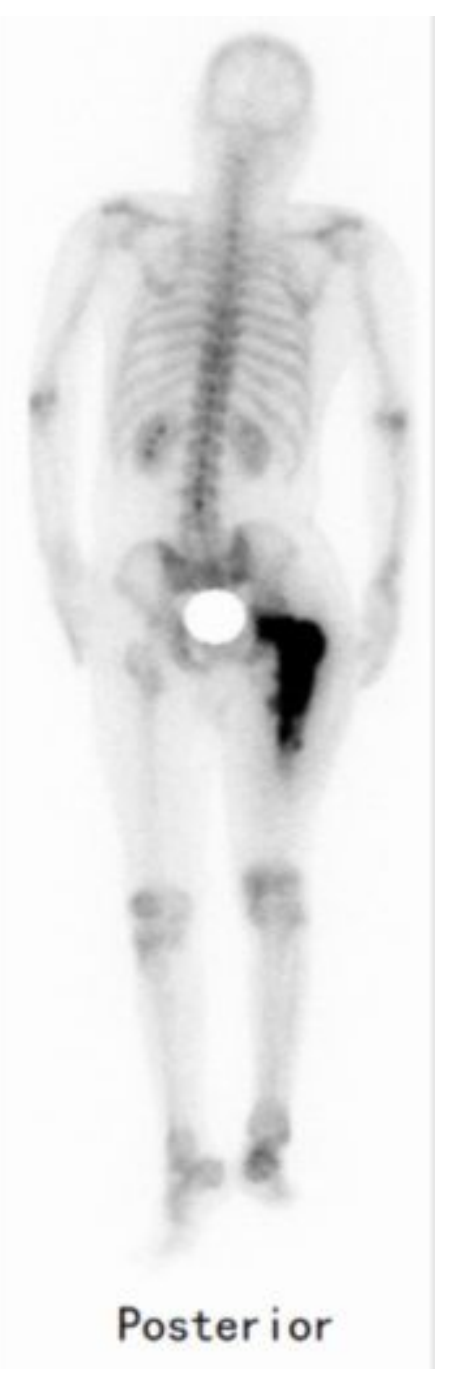

Posterior

\section{Figure 4}

Systemic bone imaging was performed 4 months after surgery. The results showed an abnormal concentration of radionuclides in the proximal region of the right femur, which was considered tumor bone metastasis.
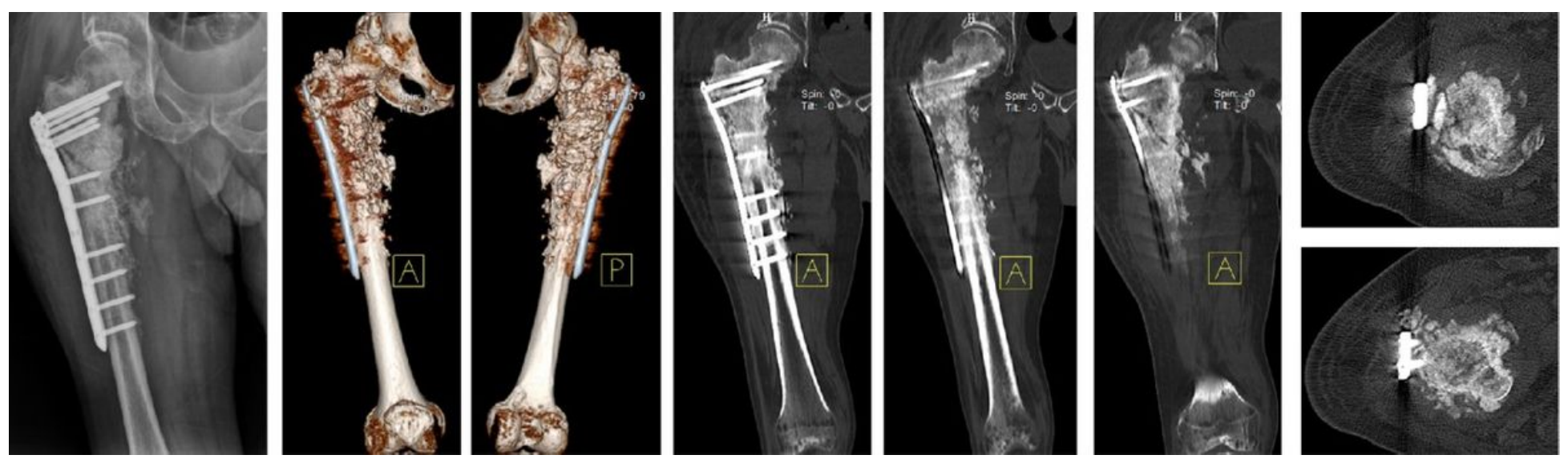

Figure 5 
Nine months after surgery, the tumor growth in the proximal femur region was completely out of control. A large amount of abnormal osteogenesis occurred, the femoral trochanter was completely dissolved, and the fracture line was clearly visible; at the horizontal plane, CT revealed that the tumor had completely penetrated the bone cortex.
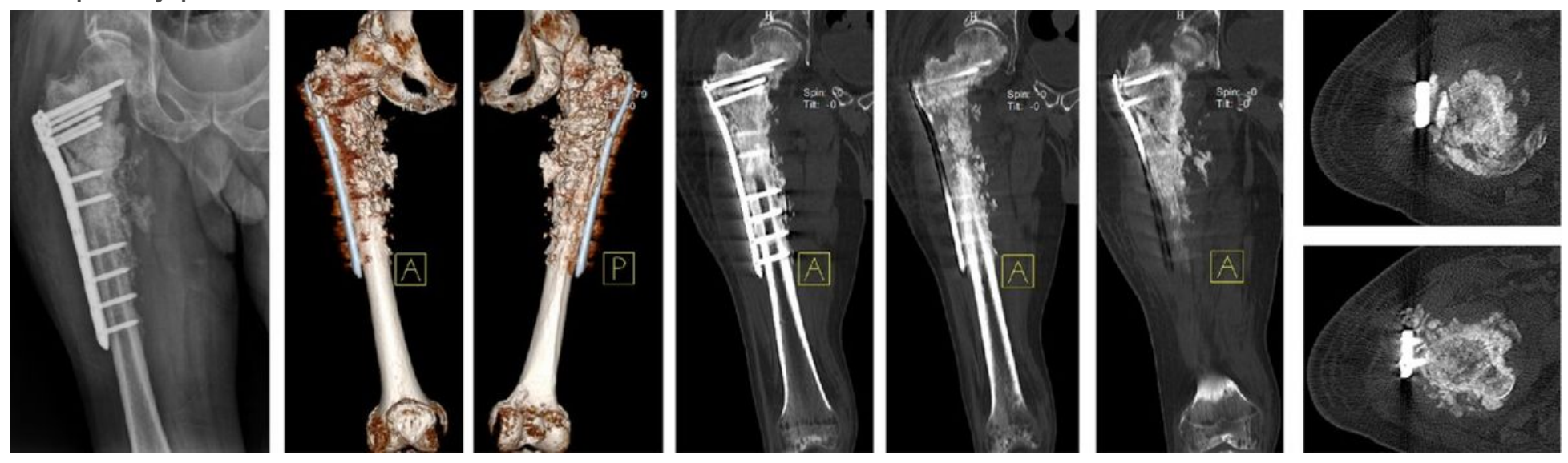

\section{Figure 5}

Nine months after surgery, the tumor growth in the proximal femur region was completely out of control. A large amount of abnormal osteogenesis occurred, the femoral trochanter was completely dissolved, and the fracture line was clearly visible; at the horizontal plane, CT revealed that the tumor had completely penetrated the bone cortex.
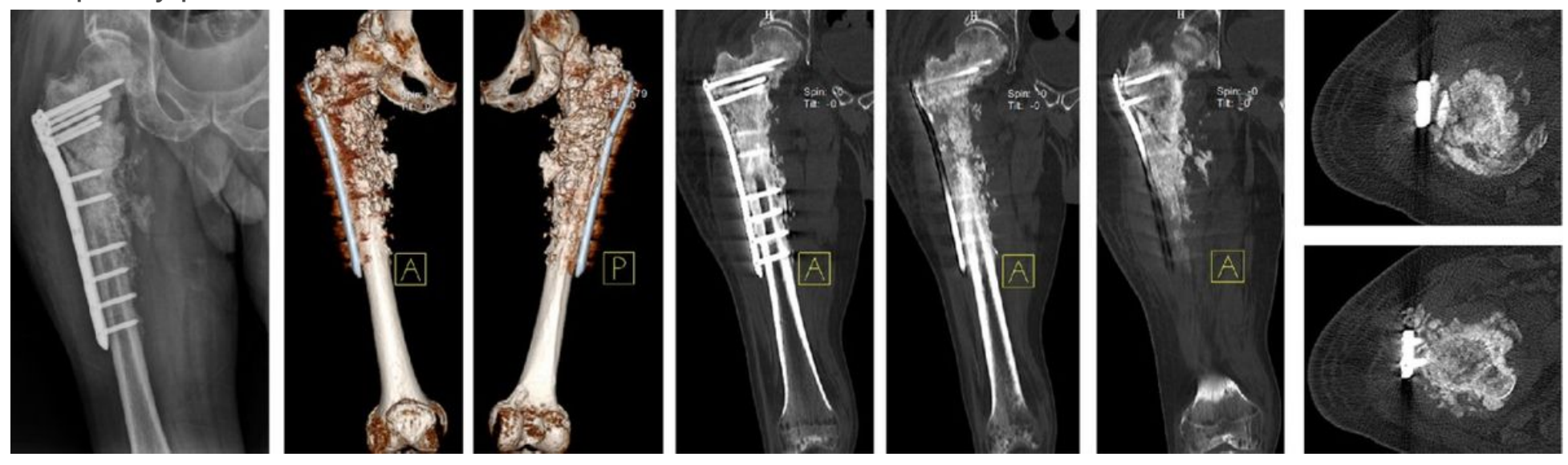

\section{Figure 5}

Nine months after surgery, the tumor growth in the proximal femur region was completely out of control. A large amount of abnormal osteogenesis occurred, the femoral trochanter was completely dissolved, and the fracture line was clearly visible; at the horizontal plane, CT revealed that the tumor had completely penetrated the bone cortex. 

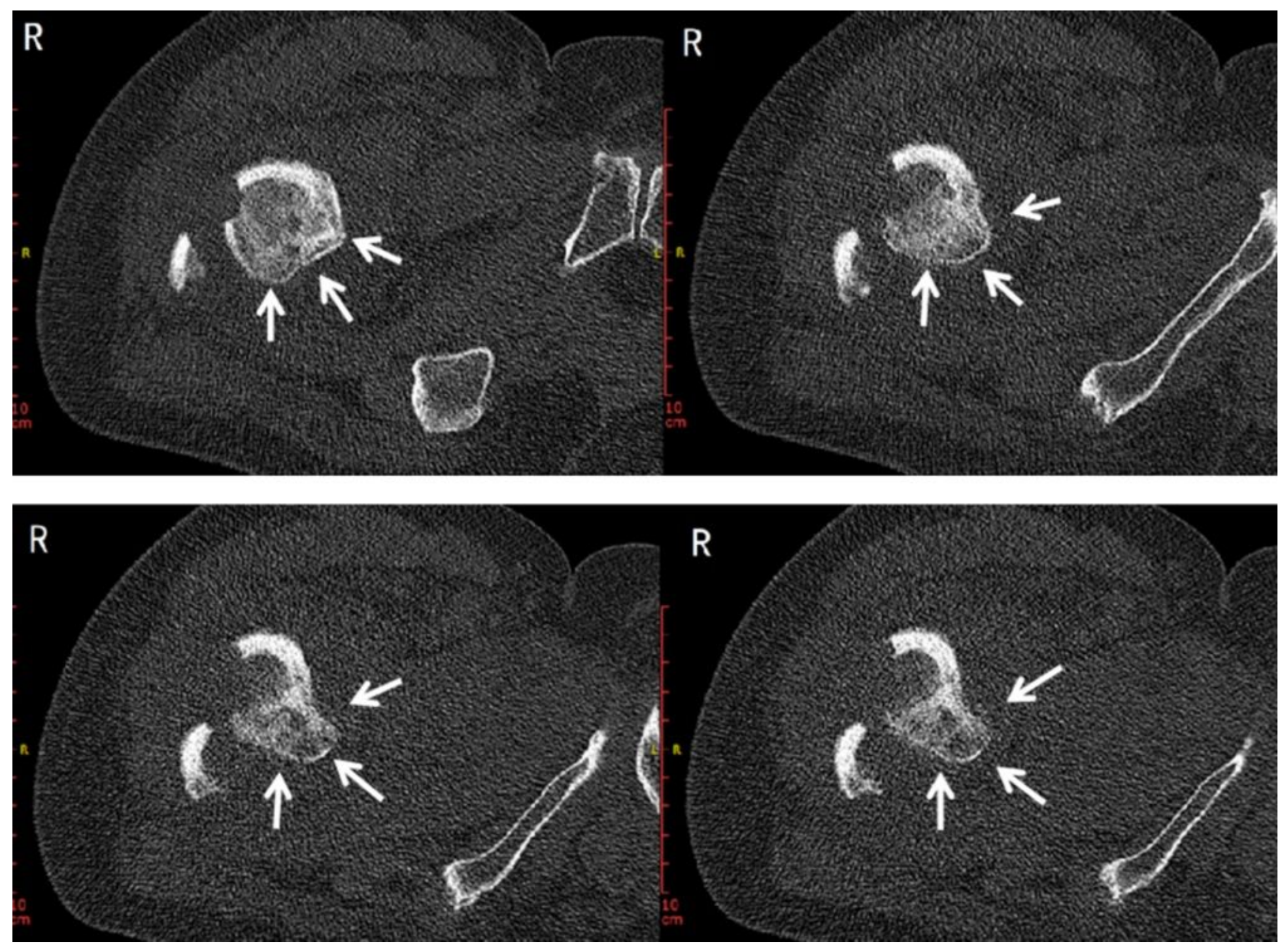

Figure 6

The patient's CT examination after his fall was retrospectively analyzed; at the bottom of the femur fracture zones, the bone cortex appeared to dissolve at the 2 o 'clock to 8 o 'clock directions, and the small rotor area had both osteolytic and osteoblastic lesions, resulting from bone destruction caused by bone metastases of colorectal cancer; thus, this is a pathological fracture. 

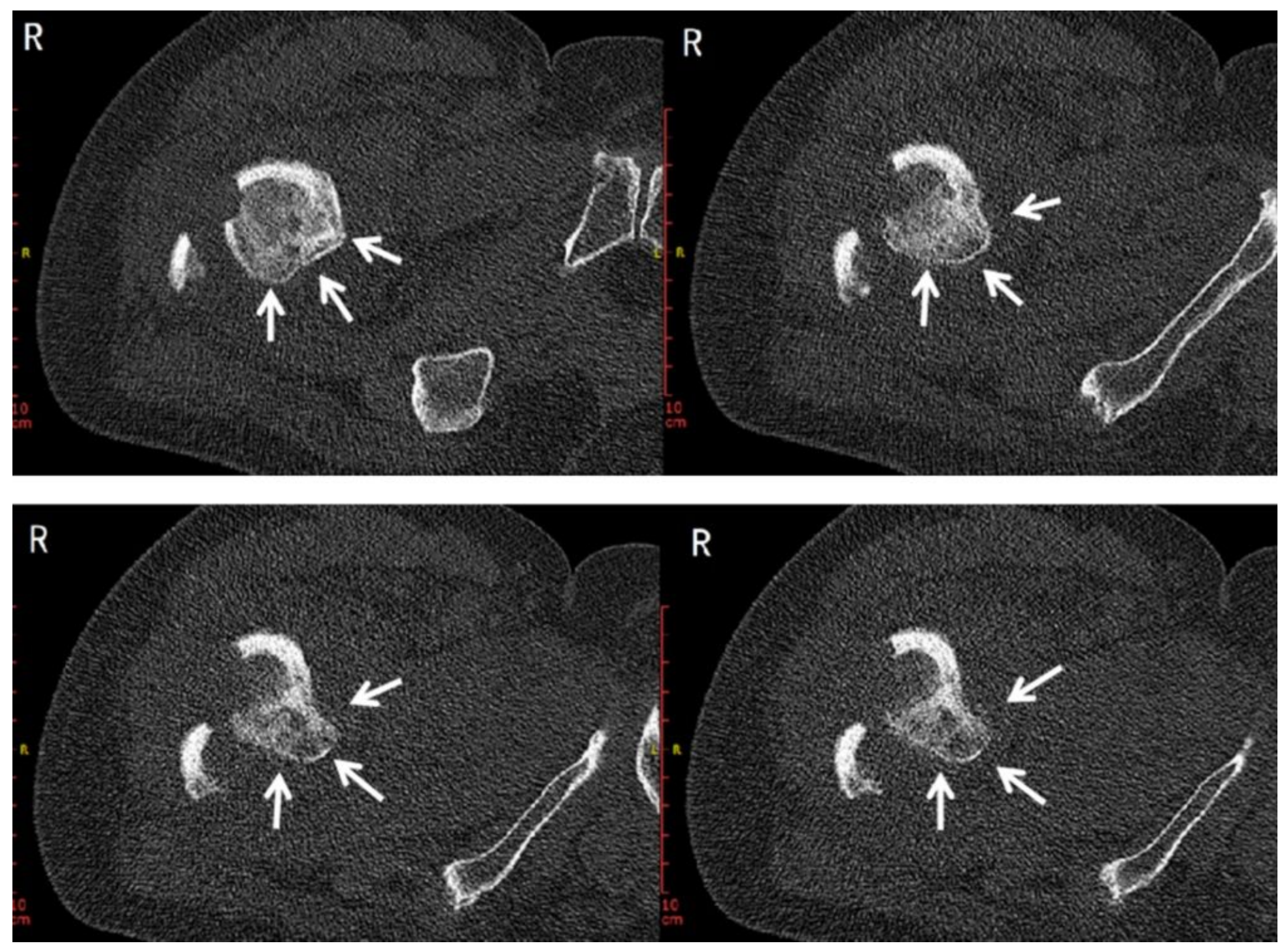

Figure 6

The patient's CT examination after his fall was retrospectively analyzed; at the bottom of the femur fracture zones, the bone cortex appeared to dissolve at the 2 o 'clock to 8 o 'clock directions, and the small rotor area had both osteolytic and osteoblastic lesions, resulting from bone destruction caused by bone metastases of colorectal cancer; thus, this is a pathological fracture. 

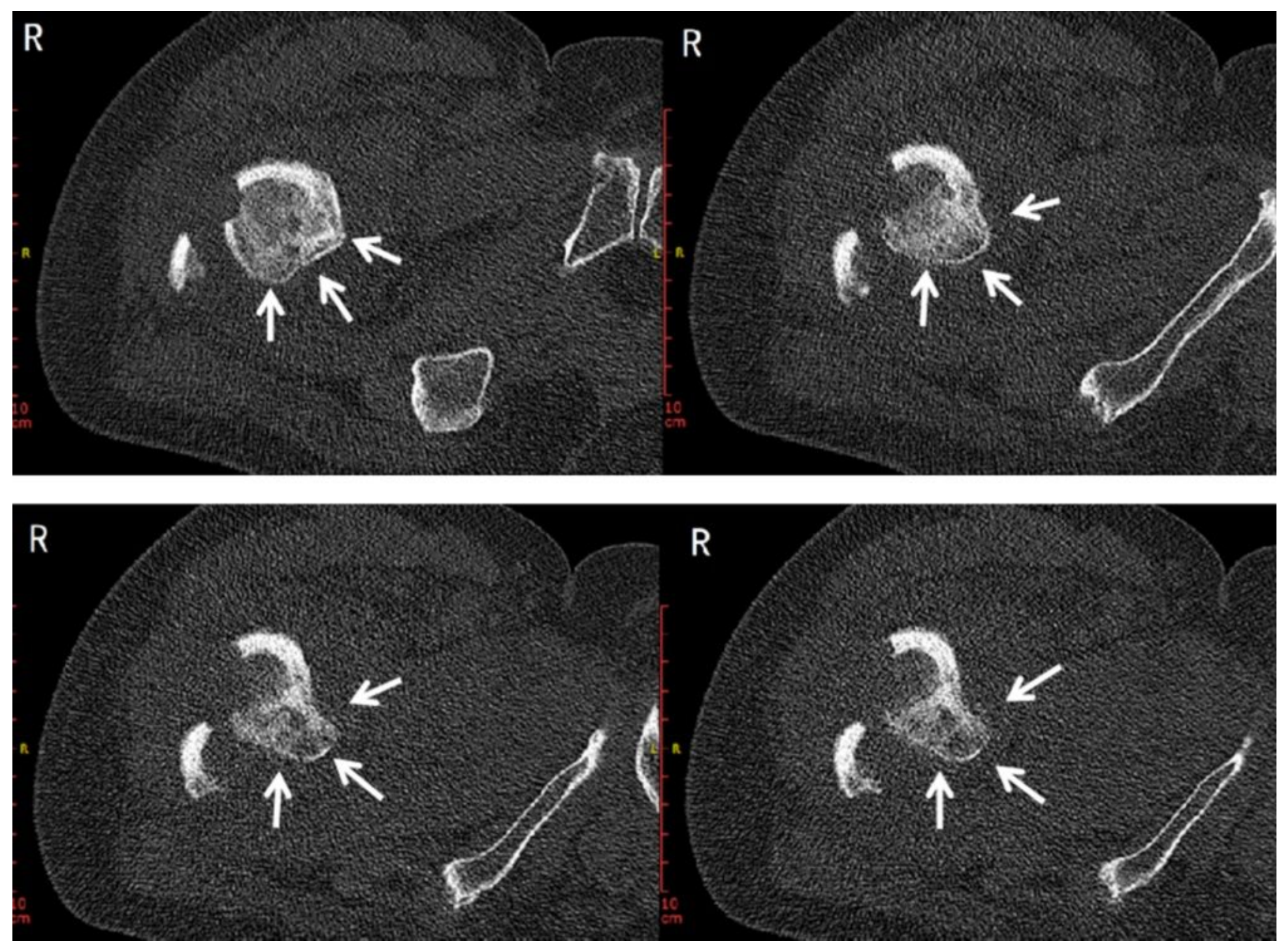

Figure 6

The patient's CT examination after his fall was retrospectively analyzed; at the bottom of the femur fracture zones, the bone cortex appeared to dissolve at the 2 o 'clock to 8 o 'clock directions, and the small rotor area had both osteolytic and osteoblastic lesions, resulting from bone destruction caused by bone metastases of colorectal cancer; thus, this is a pathological fracture. 